\title{
A Survey of Major Challenges and Future Directions for Next Generation Pervasive Computing
}

\author{
Yong-Bin Kang ${ }^{1}$ and Yusuf Pisan ${ }^{2}$ \\ 1 Institute for Graphics Interfaces \\ 3rd Fl, Ewha-SK Telecom Bldg. 11-1, Daehyun-dong, Seodaemun-gu, Seoul 120-750, \\ Korea \\ ybkang@igi.re.kr \\ 2 Department of Software Engineering \\ Faculty of Information Technology \\ University of Technology, Sydney \\ PO Box 123, Broadway NSW 2007, Australia \\ ypisan@it.uts.edu.au
}

\begin{abstract}
Pervasive computing has emerged as a new computing paradigm with a great deal with appeal in our everyday environment. However, the benefits offered by this new computing paradigm are relatively below our expected standard. This paper discusses the major challenges for the next generation pervasive computing and the difficulties in developing a promising system to meet these challenges. Then, we present a survey that covers relevant existing approaches addressed to overcome the challenges. Finally, we highlight future research directions and present a new intriguing exploration, aiming to broaden the appeal and bridge the gap for the fulfillment of the challenges.
\end{abstract}

\section{Introduction}

This paper discusses some of the major challenges for developing a promisingly user-centric computing system, and highlights the future research directions which are yet to be properly addressed to overcome the challenges in a highly embedded interactive computing space.

The development of computing technologies with the advent of a variety of computing devices and applications have been augmenting our everyday environment, seamlessly integrating our activities into the environment. This trend leads to promote a new paradigm of computing, called pervasive computing[1].

One of the principle goals of pervasive computing is to be user-centric, providing computational support to help and mediate user activities in a natural, non intrusive way $[2,1]$.

Yet, accomplishing that goal is broad and still largely undeveloped. In order to understand the difficulties to realize that goal, this paper aims at (1) classifying the major three challenges; (2) reviewing some of the major current approaches to meet the challenges; (3) presenting future research directions and a new promising exploration. 


\subsection{Key Terms}

To help understanding of this paper, some key terms are defined as follows[3, 4 , 1]:

- Context: Any information that can be used to characterize the situation of an entity, where an entity can be a user, place, or physical or computational object.

- Service: This denotes a context-aware service. It provides relevant information to users, where relevancy depends on users activities and preferences.

- Pervasive Computing: This computing paradigm focuses on augmenting the everyday environment with computing systems. It puts forth a vision of people and environments augmented with computational resources that provide information and services when and where desired.

- Pervasive computing environment (PCE): This environment is populated by a collection of users, computational resources, and the services. This environment can accommodate the pervasive computing paradigm. In this paper, we call it simply as $P C E$.

- Pervasive computing system: This system has an ability to capture and analyze the context and relationships between user activities and the services. It has to be capable of realizing the pervasive computing paradigm.

This paper is organized as follows. In the next section, we highlight the key challenges that need to be addressed. Section 3 reviews related work. In section 4 , our future directions are presented. Finally conclusion remarks close this paper.

\section{Key Challenges}

We highlight the key challenges that need to be addressed to develop a next generation system for pervasive computing based on our observations. These are classified into three major issues and each one is closely related to the others.

\subsection{Challenge 1: How to model context information}

First and foremost, modeling context information is a significant challenge. Main reasons are considered as follows:

- Complex Structure: Most PCEs may form an enormously complex structure consisted of a wide variety of heterogeneous context information $[5,6]$.

- Dynamic Environment: The context in a PCE cannot be represented by a fixed prearranged plan, since it is continuously ever-increasing and everchanging by means of (1) diverse interactions among context; (2) the numerous details that might not be noticed at design time of the PCE[7-9].

- Difficulty in incorporating user preferences: A true user-centric system needs to encapsulate user preferences into a PCE. However, capturing and understanding the user preferences that may shift over time are a quite difficult problem[10,6]. 


\subsection{Challenge 2: How to understand complex interactions}

One of the greatest challenges of pervasive computing is to capture the current status of PCE to support and enhance the user activities in execution task. For this, we believe that diverse interactions happened in PCE must be accurately detected and understood.

There can be occurred several types of interactions in PCE as follows[1113]: (1) between users and the environment; (2) among multiple environmental resources; (3) among multiple users; (4) among small social groups(e.g., family, friend); (5) different combination of those types.

Understanding those interactions is a quite difficult challenge due to the following reasons:

- Not explicitly identified: All the potential interactions cannot be identified by explicit human specification. We do not correctly know what a user like to do, and which services are preferred for user activities at the design time of a PCE $[13,14]$.

- Dynamic and Complicated Interactions: Detecting the dynamic interactions among a wide range of different context is also a difficult problem[15-20]. This is still ongoing research issue.

- Arbitrary Interaction: It is a common agreement that there can be existed not only the broad patterns of interactions, but also more sophisticated and arbitrary interactions in PCE

\subsection{Challenge 3: How to provide the most attractive services}

Lastly, pervasive computing is a user-centric view of computing. This means the best services must be supported to all the users in a PCE. Further, those services should not be contradicted with user preferences. For example, if the dynamic configuration is not consistent with user preferences, the user may be confused or surprised by that change[21].

We embody a minimal set of necessary challenges for this requirement as follows:

- Eliciting user preferences: First of all, in order to provide the most attractive services to the users, we must be able to elicit user preferences. Since, however, user preferences are invisible and seamlessly shift over time, it is a significant challenge[10,6].

- Providing preference resolution policies: Within a PCE, every user has different preferences or makes different decisions about the same context. This implies that a particular user might intrude or distract other's preferences intentionally or accidentally. Let us call this situation as preference confliction. Since this situation can be commonly happened in the shared environment, harmonizing that confliction is quite important $[22,12]$.

- Providing future prediction mechanism: As Weiser noted[1], the whole purpose of pervasive computing is to provide application that serve humans. For this, we believe that a pervasive computing system should be able to predict future possible actions for use conveniences at a proper place in proper time. 
- Providing collaborative services: If the interrelated services in the environment are adequately and seamlessly collaborated, we will possibly provide more intelligent services and will improve the quality of the services. We call those services as collaborative services.

\section{Review of Existing Approaches}

There has been a large body of significant research activity for pervasive computing in the last few years both in academic as well as industry. In this section, we briefly review the current status of existing approaches for resolving each challenge discussed above.

\subsection{Modeling for PCE}

It is a fundamental design question how to most appropriately design a model for a PCE being able to capture and encapsulate a large amount of information in the environment. Finding the answer of the question is quite important, because such a model provides computational support for constructing, describing, implementing, and analyzing the patterns of the interaction and coordination among context. We introduce popularly used some of the models heading for this attempt.

Semantic Network is a graph notation for representing the knowledge of the context in patterns of interacted nodes and links[23]. This network is an appropriate model for capturing a vast amount of information entering an Intelligent Environment, deployed at MIT's AI Laboratory[6]. It can encapsulate many different objects and associations, track, and follow the relationships between them.

CML is designed by Henricken[10] to capture many of the features of context information. CML provides a formal basis for representing and reasoning about some of the properties of context information. CML allows a graphical notation for describing information types, their classification, relevant quality of metadata, and dependencies among different types of information. A main benefit of CML is sufficiently generic to capture arbitrary types of context information.

Multimodal context[17] was announced to meet the requirement of broader notion in ubiquitous computing system. DAML and DAML+OIL[24], and OWL[25] are ontologies that are a kind of classification for expressing the entities which consist of environment based on W3C's Resource Description Framework(RDF) [26]. Robinson[27] proposes a middleware language that aid in service composition in pervasive computing. It brings together and applying research in the field of Natural Language Processing(NLP), Human Computer Interaction(HCI) and Network Service[28]. Coen[29] introduces a natural language interface to a highly interactive space, known as the Intelligent Room.

Most of these models are required to be formulated by human explicit specification at design time of their PCEs. Thus, those are appropriate for expressing the environment in simply static structure. 
However, because of the diversity and the dynamics that is expected in a PCE, those modeling methods can be far less suitable to represent a dynamic PCE involving sophisticated interactions. Furthermore, the PCE is everchanging, even the characteristics of the context are unpredictable (e.g., rapid, unbounded, and time-varying), and thus those are ill-suited to meet the challenges.

\subsection{Understanding Interactions in the Environment}

In order to capture the diverse interactions in a PCE, several middleware systems have been developed.

In PACE Project[30], a middleware was developed for distributed contextaware systems and analyzed the state-of-the-art in middleware for context-aware systems. The PACE consists of four main subsystems such as a context management system, a preference management system, a programming toolkit that facilitates interaction between users and environment, and communication assistance tools.

Dey[17] and Pervasive Computing Group[8] propose middleware systems for detecting the current state, minimizing user distractions. Grimm[31] and Song[32] provide frameworks that can make users simply and easily access services. Mani[13] provides interaction paradigm that utilizes the full potential of the networked, multimedia environment.

Most of these requires the users to pre-arrange the relationships of the interactions between the users and the environment. For example, the one wish to participate in the shared environment must construct and combine their own relationships of interactions. In addition, most interpretation of those interactions are performed in the limited bounds of spaces. Furthermore, those approaches have an inability to dynamically reconfigure the relationships of the interactions. As a result, understanding the interactions in a PCE is still needed to be developed and is a difficult research issue.

\subsection{Providing the best services to the users}

Very few attempts addressing the issue about improving technique for dynamically eliciting user preferences according to temporal changes has been studied until recently in our observations. Most have been attempted based on policybased method[33, 12,34]. The policy-based method allows users to formulate their own preferences by explicit policies if necessary. However, this is only useful at design time, thus it cannot incorporate the dynamic changes of user preferences. Once formulated, these preferences will be gradually staled with the lapse of time, since preferences may shift over time.

We now introduce the representative three models of the policy-based method. Composite Capabilities/Preference Profiles (CC/PP) [35] is a W3C proposed standard [36] for describing device capabilities and preferences with a focus on wireless devices such PDAs and mobile phones. It is suitable for expressing very simple preferences, allowing a system to customize preferences. However, future fledged 
pervasive systems will require much more sophisticated preferences in order to support seamless adaption to changes in these preferences. Thus, this model is not suitable for future pervasive systems. A more natural preference modeling method based on situation abstraction is developed by Henricksen[22]. In this work, users can express a set of simple and possibly conflicting preferences. In addition, preferences can be grouped into sets and combined by policies. The confliction detection model by Pervasive Computing Group[8] is introduced. This model presents the concept of interest zone which is main basis for conflicting resolution model based on user behavioral patterns.

Regarding the future prediction technique, there has been a large body of research about predicting future situations in static structured spaces. One of the major common features is that these approaches need prior knowledge about their information space. For examples, Chen[37] proposes a prediction model for navigating category on web by inferencing the user access patterns. Letizia[38] and Chi[39] infer user interests from on the web. Yan[40] and $\mathrm{Fu}[41]$ cluster the users based on the preference from on the web. Lam[42] proposes a Bayesian approach to track user interest shift.

However, very little research has been performed to predict promising future states of dynamic computing environments. Besides, no attempts to provide intelligent collaborative services for pervasive computing have been addressed until now based on our investigation.

\subsection{Major Case Studies}

It is helpful to briefly review what major case studies have been implemented to realize the PCE. Aura Project[18], Intelligent Room[11], Aware Home[19], and EasyLiving[15] have been explored the intelligent environment that can allow people to interact with computing systems.

For example, Aura focuses on the pervasive distraction-free computing and investigates the system and network level adaptation issues required for a PCE. However, there is not much of a focus on the user interaction with media.

The Aware Home project attempts to create a home environment that is aware of its user activities and locations. This environment is a set of a number of building blocks consist of indoor location tracking, activity recognition, largescale projective displays, wireless sensor networks, etc. However the context is limited to answering a limited set of questions related to the user such as where, what and who. The system continuously monitors user activities to get more information about the user to build the context.

Intelligent Room project describes criteria for creating highly embedded, interactive space with multiple vision and speech based sensing technologies.

The common features of these projects focus on the attempts to support distraction-free computing environments where people can perform their tasks without interventions by the system and environment.

However, in these projects, understanding the interactions in the environment can be made by some extent of explicit definition in limited bounds of 
spaces. In addition, those still fail to address the question of the new interactions when the new constituents are involved into the PCEs, the implication of it. Furthermore, no attempts to dynamically elicit user preferences and to provide the collaborative services are addressed.

Therefore, we need new approaches to design the next generation pervasive computing system that will be able to resolve those problems.

\section{Future Directions}

This section briefly describes future directions to design a next generation pervasive computing system on the basis of the discussed challenges.

\subsection{How to model context information}

First, we believe that we need a new model that has a formless schema for a PCE that will not be limited to a particular environment. This model must be able to spontaneously and seamlessly evolve its structure by (1) self-learning from interaction patterns; (2) self-configuring the environment; and (3) self-inferring the future states, according to the environmental changes without human control.

Second, we expect that uniform communication languages and standardized ontologies will be essential, in order to provide seamless inter-operability and ease of the evolution of a PCE.

Third, we believe that a technique for formalizing user preferences into a appropriate model must also be presented.

\subsection{How to understand complex interactions}

First, we need uniform interfaces to provide single paradigm of data delivery for promoting complex context interactions.

Second, based on this interface, we believe that techniques for capturing, organizing, and maintaining the snapshot of the active states of the environment such as user activities or preferences must be developed.

Third, we expect that a mechanism for identifying similar groups (community) based on their activities and preferences should be presented, in order not to distract other group members' preferences.

\subsection{How to provide most attractive services}

First, in order to elicit user preferences, we believe that a promising criteria for learning the current behavioral patterns of a user need to developed. Because, people usually perform their activities under the influence of their preferences consciously or unconsciously.

Second, we believe that a computing technique for the similarity or the dissimilarity measure about the individual preferences must be developed, in order to resolve the preference confliction situations. 
Third, a new prediction mechanism based on analysis of the prior knowledge about user's previous patterns will be needed.

Last, a middleware language and a standard mechanism that aid in incorporating services in pervasive computing must be designed. These services should be fully cooperated all the potential of the computing environment, integrating and manipulating services seamlessly.

\subsection{Where can we find promising possibilities}

How can pervasive computing simultaneously broaden its appeal and effectively cope with the future research issues noted above?

To answer that question, we present a new intriguing exploration to find a new promising potential by means of investigating the similarities between a biological space and a PCE.

Bioinformatics has arisen from the needs of biologists to utilize and help interpretation the vast amount of data that are constantly being gathered in genomic research [43]. More specifically, we have found that the following common challenges that must be shed light on in human living system are shared in bioinformatics, as well as the biological world has been remarkably successful in solving these problems. [5, 43-45]:

- How to model a genetic network: Human living system forms a huge genetic network composed of non-identical elements (e.g., genes and proteins). Biologists need a new paradigm that is reliable and can deal with the network whose nodes are genes and proteins, and the chemical interactions represented by edges between them. To govern such a constantly expanded large genetic network, a new robust self-organization model is needed. Equally complex situation is occurred in a PCE, where a vertices are diverse entities(e.g., person, devices, and services) and edges are the interactions between them.

Thus, we expect if we more focus on finding the detailed common characteristics from detailed data has been available, and examining generic properties between those two spaces, we will formalize the existence of high-degree of self-organization model far beyond our expectation.

- How to enlighten the relationships among genes: Enlightening internal/external relationships among genes in DNA that contains millions of symbols is also a major focus area in biological space. Genes are usually represented by Strings to represent DNA, RNA, and sequences of amino acids. Each DNA string contains millions of symbol and metabolic that describes the behavior of cells and their interactions with the network environment.

Some of the most important algorithmic trends in bioinformatics is to study of the relationships among genes such as (1) finding similarities among strings; (2) detecting certain patterns within strings; (3) finding similarities among parts of spatial structures. For these, the remarkable developments of pattern-matching approach has been developed since 1990s. For examples, Basic Local Alignment Search Tool (BLAST) that mimics the behavior of 
the dynamic programming approach and efficiently yields good results. Another widely used and effective technique is multiple alignment, which helps align several sequences of symbols, so identical symbols are properly lined up.

Therefore, we believe that learning and using those techniques into the computing space of PCEs will be very helpful to detecting, capturing, and analyzing the interactions mechanisms in PCEs.

- How to infer new behavior patterns: Inferring new behavior patterns of biological data according to the previous classified clustering data is also one of the major research issues in bioinformatics. Biological data is often characterized by huge size, duplication, unavailability, and sometime unreliability. For this fact, inferring possible sequences from existing data is most essential in biological world. There is much of research about inferring sequences of genes from typical sequences and establishing the likelihood of the patterns for future sequences.

We believe that such inferring techniques will be effectively utilized into the situation requiring dynamic prediction techniques of possible future states(e.g., user activities, preferred services) in PCEs.

The biological world has been remarkably successful in problem solving of the evolution techniques of organisms, the analysis of complex arbitrary interactions, the inference about possible functions, and even cooperative behaviors among the elements in a enormous genetic network. Therefore, we believe that the employment of relevant research topics in bioinformatics will be eventually helpful to find new promising possibilities about the discussed challenges in this paper.

\section{Conclusion}

In this paper, we briefly discussed some of the important challenges, explored the existing approaches and presented the future research direction issued for developing user-centric pervasive computing.

More specifically, we highlighted the following three challenges that need to be addressed: (1) how to correctly model context information; (2) how to understand the interactions happened in PCEs; (3) how to provide the best services satisfying user preferences. Then, we reviewed some of the relevant existing approaches and their limitations to meet the challenges. Finally, we generated future research directions for realizing the next generation pervasive computing based on our observations. In particular, we suggested the promising possibilities of the involvements of commonly shared topics in bioinformatics, and briefly discussed common features between the biology space and the pervasive computing space.

We hope the issues addressed in this paper will both stimulate further discussion and be more helpful to bridge the gap in a further step towards the next generation pervasive computing technology 


\section{References}

1. Weiser, M.: The computer of the twenty-first century. Scientific American 3 (1991) 66-75

2. Gupta, S., W.C.Lee, Purakayastha, A., Srimani, P.: An overview of pervasive computing. IEEE Personal Communications 4 (2001) 8-9

3. Dey, A., Abowd, G., Salber, D.: A context-based infrastructure for smart environments. Proceedings of the 1st International Workshop on Managing Interactions in Smart Environments (MANSE '99) (1999) 114-129

4. Sheng, Q.Z., Benatallah, B.: Contextuml - a uml-based modeling language for model-driven development of context-aware web services. Proceedings of the International Conference on Mobile Business (2005) 206-212

5. Barabasi, A., Albert, R.: Emergence of scaling in random netowork. Science 286 (1999) 509-512

6. Peters, S., Shrobe, H.: Using semantic networks for knowledge representation in an intelligent environment. Proceedings of the IEEE International Conference on Pervasive Computing and Communications (2003) 323-329

7. Cetintemel, U., Franklin, M.J., Giles, C.L.: Self-adaptive user profiles for largescale data delivery. In Proc. of the 16th International Conf. on Data Engineering (2000) 622-633

8. Group, P.C.: Middleware infrastructure for active surroundings. Technical Report TR-CSPG-04-28-001, Information and Communication University (2003)

9. Nelson, G.J.: Context-aware and location systems. A dissertation submitted for the degree of $\mathrm{PhD}$ in the University of Cambridge (1998)

10. HENRICKSEN, K., INDULSKA, J., RAKOTONIRAINY, A.: Modeling context information in pervasive computing systems. Proc. of the First International Conference on Pervasive Computing (2002) 15-26

11. Hanssens, N., Kulkarni, A., Tuchida, R., Horton, T.: Building agent-based intelligent workspaces. ABA Conference Proceedings (2002) 675-681

12. Lee, D., Hyun, S.J., Lee, Y.H., Lee, G., Han, S., Kang, S.H., Park, I., Choi, J.: Active surroundings - a group-aware middleware for ubiquitous computing environments. Ubiquitous Computing Workshop 2004 (2004)

13. Mani, A., Sundaram, H., Birchfield, D., Qian, G.: The networked home as a usercentric multimedia system. NRBC '04 - Proceedings of the 2004 ACM workshop on Next-generation residential broadband challenges (2004) 19-30

14. O'Sullivan, D., Lewis, D.: Semantically driven service interoperability for pervasive computing. Proceedings of the 3rd ACM international workshop on Data engineering for wireless and mobile access (2003) 17-24

15. Brumitt, B., Meyers, B., Krumm, J., Kern, A., Shafer, S.A.: Easyliving - technologies for intelligent environments. Proceedings of the 2 nd international symposium on Handheld and Ubiquitous Computing (2000) 12-29

16. Coen, M.H.: Design principles for intelligent envrionments. In Proc. of the 1998 National Conference on Artificial Intelligence (AAAI-98) (1998) 37-43

17. Dey, A., Salber, D., Abowd, G.: A conceptual framework and a toolkit for supporting the rapid prototyping of context-aware applications. Human-Computer Interaction 16 (2001) 97-166

18. Garlan, D., Siewiorek, D., Smailagic, A., Steenkiste, P.: Project aura - toward distraction-free pervasive computing. IEEE Pervasive Computing 2 (2002) 22-31

19. Kidd, C., Orr, R., Abowd, G., Atkeson, C., Essa, I., MacIntyre, B., Mynatt, E., Starner, T., Newstetter, W.: The aware home - a living laboratory for ubiquitous 
computing research. Proceedings of the Second International Workshop on Cooperative Buildings, Integrating Information, Organization, and Architecture (1999) 191-198

20. Kulkarni, A.: Design principles of a reactive behavioral system for the intelligent room. in Bitstream - The MIT Journal of EECS Student Research (2002)

21. Nakajima, T., Fujinami, K., Tokunaga, E., Ishikawa, H.: Middleware design issues for ubiquitous computing. Proceedings of the 3rd international conference on Mobile and ubiquitous multimedia (2004) 55-62

22. HENRICKSEN, K., INDULSKA, J.: A software engineering framework for context-aware pervasive computing. Proc. of the Second IEEE International Conference on Pervasive Computing and Communications (2004) 77-86

23. Sowa, J.F.: Semantic networks. Encyclopedia of Artificial Intelligence (1992)

24. DAML: Darpa markup language. www.daml.org (2003)

25. Dean, M., Connolly, D., van Harmelen, F., Hendler, J., Horrocks, I., McGuinness, D.L., Patel-Schneider, P.F., (editors), L.A.S.: Owl web ontology language 1.0 reference. http://www.w3.org/TR/owl-ref/ (2002)

26. Brickley, D., Guha, R.V.: Resource description framework (rdf) schema specification 1.0 - rdf schema. W3C Working Draft (2003)

27. Robinson, J., Wakeman, I., Owen, T.: Scooby - middleware for service composition in pervasive computing. Proceedings of the 2nd workshop on Middleware for pervasive and ad-hoc computing 77 (2004) 161-166

28. Weeds, J., Keller, B., Weir, D., Wakeman, I., Rimmer, J., Owen, T.: Natural language expression of user policies in pervasive computing environments. Proceedings of OntoLex 2004 (LREC Workshop on Ontologies and Lexical Resources in Distributed Environments (2004)

29. Coen, M., Weisman, L., Thomas, K., Groh, M.: A context sensitive natural language modality for the intelligent room. Proceedings of MANSE'99 (1999)

30. HENRICKSEN, K., INDULSKA, J.: Developing context-aware pervasive computing applications - models and approach. Pervasive and Mobile Computing 2 (2005) $37-64$

31. Grimm, R.: System support for pervasive applications. ACM Transactions on Computer Systems 22 (2004) 421-486

32. Song, Z., M., R., Agre, J., Labrou, Y.: Task computing for ubiquitous multimedia services. Proceedings of the 3rd international conference on Mobile and ubiquitous multimedia (2004) 257-262

33. Dunlop, N., Indulska, J., Raymond, K.: Methods for conflict resolution in policybased management systems. In Proc. of the 7th IEEE International Enterprise Distributed Object Computing Conference (EDOC03) (2003) 98-111

34. Wang, B., Bodily, J., Gupta, S.K.: Supporting persistent social group in ubiquitous computing environments using context-aware ephemeral group service. Proceedings of Pervasive Computing and Commnunications(PERCOM04) (2004) 287-296

35. INDULSKA, J., ROBINSON, R., RAKOTONIRAINY, A., HENRICKSEN, K.: Experiences in using cc/pp in context-aware systems. International Conference on Mobile Data Management (2003) Springer Verlag, LNCS 2574, 247-261

36. F., R., C., W., H., O., G., K.: Composite capability/preference profiles(cc/pp) structure and vocabularies. W3C Working Draft (2001)

37. M.Chen, LaPaugh, A., Singh, J.: Predicting category accesses for a user in a structured information space. Proceedings of the 25th annual international ACM SIGIR conference on Research and development in information retrieval (2002) $65-72$ 
38. Lieberman, Letizia, H.: An agent that assists web browsing. Proceedings of the 1995 International Joint Conference on Artificial Intelligent (1995) 65-72

39. Chi, E.H., Pirolli, P., Chen, K., Pitkow, J.: Using information scent to model user information needs and actions and the web. Proceedings of the SIGCHI conference on Human factors in computing systems (2001) 490-497

40. Yan, T.W., Jacobsen, M., Garcia-Molina, H., Dayal, U.: From user access patterns to dynamic hypertext linking. Proceedings of the fifth international World Wide Web conference on Computer networks and ISDN systems (1996) 1007-1014

41. Fu, Y., Sandhu, K., , Shih, M.: Fast clustering of web users based on navigation patterns. World Multiconference on Systemics, Cybernetics and Informatics (SCI/ISAS'99) 5 (1999) 560-567

42. Lam, W., Mostafa, J.: Modeling user interest shift using a bayesian approach. Journal of the American Society for Information Science and Technology 5 (2001) 416-429

43. Cohen, J.: Bioinformatics-an introduction for computer scientits. ACM Computing Surveys 36 (2004) 122-158

44. Cohen, J.: Computer science and bioinformatics. COMMUNICATION OF THE ACM 48 (2005) 72-78

45. Hernandez, T., Kambhampati, S.: Integration of biological sources - current systems and challegens ahead. SIGMOD Record 33 (2004) 51-60 